\title{
Altered Biological Potential and Radioresponse of Murine Tumors in Different Microenvironments
}

\author{
Ik Jae Lee, MD, $P h D^{1}$ \\ Eun Jeong Lee, $\mathrm{PhD}{ }^{1}$ \\ Hyojin Park, PhD² \\ Wonwoo Kim, $\mathrm{PhD}^{3}$ \\ Sang-Jun $\mathrm{Ha}, \mathrm{PhD}^{2}$ \\ You Keun Shin, BSc ${ }^{4}$ \\ Jinsil Seong, MD, $P h D^{1}$
}

\section{${ }^{1}$ Department of Radiation Oncology, Yonsei University College of Medicine, Seoul, ${ }^{2}$ Department of Biochemistry, \\ College of Life Science and Biotechnology, \\ Yonsei University, Seoul, \\ ${ }^{3}$ Department of Radiation Treatment Research, Korea Institute of Radiological and \\ Medical Sciences, Seoul, \\ ${ }^{4}$ Cancer Metastasis Research Center, Yonsei Institute for Cancer Research, Seoul, Korea}

Correspondence: Jinsil Seong, MD, PhD

Department of Radiation Oncology,

Yonsei University College of Medicine,

50 Yonsei-ro, Seodaemun-gu, Seoul 03722, Korea

Tel: 82-2-2228-8095

Fax: 82-2-2227-7823

E-mail: jsseong@yuhs.ac

Received December 11, 2014

Accepted June 18, 2015

Published Online July 28, 2015

*This study was presented at the 53th annual meeting of the American Society for Therapeutic Radiation Oncology (ASTRO) held October 2-4, 2011 in Miami, FL.

\section{Purpose}

This study was conducted to evaluate the biological features of murine hepatocarcinoma according to different tumor microenvironmental models and to determine the change in molecular and immunologic responses after radiation.

\section{Materials and Methods}

Tumor models were established in the liver (orthotopic) and thigh (heterotopic) of male $\mathrm{C} 3 \mathrm{H} / \mathrm{HeN}$ mice. Tumor growth and lung metastasis were assessed in these models. To evaluate the radiation effect, the tumors were irradiated with $10 \mathrm{~Gy}$. Factors associated with tumor microenvironment including vascular endothelial growth factor (VEGF), cyclooxygenase-2 (COX-2), transforming growth factor beta1 (TGF- $\beta 1$ ), CD31, and serum interleukin6 (IL-6) were evaluated. Tumor-infiltrating regulatory immune cells, regulatory T cells (Tregs), and myeloid-derived suppressor cells (MDSCs) were also analyzed.

\section{Results}

A higher number of lung metastases were observed in the orthotopic tumor model than in the heterotopic tumor model. VEGF, CD31, COX-2, and TGF- $\beta 1$ expression was more prominent in the orthotopic tumor model than in the heterotopic tumor model. Expression of the angiogenic factor VEGF and key regulatory molecules (TGF- $\beta 1$ and COX-2) decreased following radiation in the orthotopic tumor model, while the serum IL-6 level increased after radiation. In the orthotopic tumor model, the number of both Tregs and MDSCs in the tumor burden decreased after radiation.

\section{Conclusion}

The orthotopic tumor model showed higher metastatic potential and more aggressive molecular features than the heterotopic tumor model. These findings suggest that the orthotopic tumor mouse model may be more reflective of the tumor microenvironment and suitable for use in the translational research of radiation treatment.

\section{Key words}

Tumor microenvironment, Hepatocarcinoma, Radiation

\section{Introduction}

The tumor microenvironment (TME) and surrounding adjacent normal tissues might be important modulators of radiation therapy. The TME, a complex comprising the extra- cellular matrix and various cell types, including carcinoma cells, endothelial cells, fibroblasts, and immune cells, as well as extracellular matrix molecules, is known to contribute to the carcinoma process [1]. Recent studies have convincingly demonstrated that the TME might be involved in regulation of metastasis and tumor progression [2]. In this situation, a 
number of non-neoplastic cells help comprise the TME. These non-neoplastic cells play important roles in the secretion and bioavailability of pro- and anti-metastatic factors and thus may either facilitate or suppress metastasis. Cretu and Brooks [3] suggested an expanding array of components that contribute to metastasis.

Tumor implantation into the thigh or dorsal skin (heterotopic) has been used as a standard method for establishing murine tumor models. These types of models are helpful in understanding the nature of cancers and therapeutic approaches; however, they are not sufficient for the investigation of interactions of tumor cells with the surrounding microenvironment, including immunological responses. Therefore, we established a syngeneic murine hepatocarcinoma model reflecting tumor-host microenvironment and organ selectivity via orthotopic implantation. This model has several advantages over heterotopic models of hepatocarcinoma. First, this model showed similar clinical settings including tumor location, liver damage, and biochemical changes, and supports an interaction between the tumor and host, particularly between tumor cells and microenvironment. The goal of the current study was to evaluate the biological features of orthotopic murine hepatocarcinoma and compare the molecular and immunologic responses before and after radiation.

\section{Materials and Methods}

\section{In vivo tumorigenicity}

Male $\mathrm{C} 3 \mathrm{H} / \mathrm{HeN}$ mice, 7-8 weeks old, were used in the current study. Animal experiments were performed in accordance with the Animal Research Committee's Guidelines at Yonsei University Medical College, and all facilities were approved by the Association for Assessment and Accreditation of Laboratory Animal Care. A spontaneously developed murine hepatocarcinoma (HCa-I) was generously provided by Luka Milas (University of Texas M.D. Anderson Cancer Center) and used in the current study [4]. This transplantable murine tumor is a syngeneic, highly radioresistant tumor with a radiation dose yielding a 50\% tumor cure rate greater than 80 Gy [5]. This model has a metastatic potential to the lung, as well as a full host immune response, making it useful for study of the application of immunologic findings in preclinical models of cancer [6].

\section{Establishment of heterotopic and orthotopic murine tumor models}

A heterotopic tumor model (Fig. 1A) was generated by inoculating $1 \times 10^{6} \mathrm{HCa}-\mathrm{I}$ cells into the muscles of the right thighs of the mice. To evaluate changes of the tumor and normal peritumor liver by radiation, an orthotopic tumor model was established by injection of $1 \times 10^{6} \mathrm{HCa}-\mathrm{I}$ cells directly into the subcapsular parenchyma of the left lobe of the liver. To prevent tumor cell spillage, we developed a modified surgical technique, in which a piece of surgicel (Ethicon, Somerville, NJ) is attached on the liver injection site after delivery of HCa-I cells (Fig. 1B). Two weeks after tumor cell implantation, three mice for each tumor model were randomly assigned to a radiation treatment group or control group.

\section{Frequency of lung metastases associated with hetero- topic versus orthotopic murine tumors}

In HCa-I, grown in thighs, development of spontaneous lung metastasis occurs within 5-20 days after tumor implantation [7]. The mice were randomly allocated to two groups of six animals according to the site of tumor implantation (orthotopic or heterotopic). The number of lung metastatic nodules was counted for different TMEs. Mouse lungs were taken at 6,9,12, and 15 days after tumor implantation and fixed with Bouin's solution (Fig. 1C). After 1 day, the lungs were rinsed in water to remove excess Bouin's solution and the number of surface lung metastatic nodules was counted under a light polarizing microscope $(\times 4)$.

\section{Radiation administration to the orthotopic murine tumor}

Radiation was delivered to the abdomen as a single dose of 10 Gy with $320 \mathrm{KV}$ X-ray using the X-RAD 320 (Precision $X$-ray, North Branford, CT). A lead shield containing cut-out squares, $1.5 \mathrm{~cm} \times 18 \mathrm{~cm}$ in size was used to protect the head and lower limbs from radiation, and the mice were aligned for proper liver irradiation. Radiation administration was followed by tumor inoculation 2 weeks later. Irradiated orthotopic tumor samples were obtained on the first and third days after radiation. Non-irradiated orthotopic tumor samples were also obtained on the same days for comparison with the irradiated groups.

\section{Immunohistochemical staining of tumor microenviron- mental molecules}

Immunohistochemistry was performed to assess the effect of radiation on microenvironmental molecules in heterotopic and orthotopic tumors, including angiogenic factors such as 

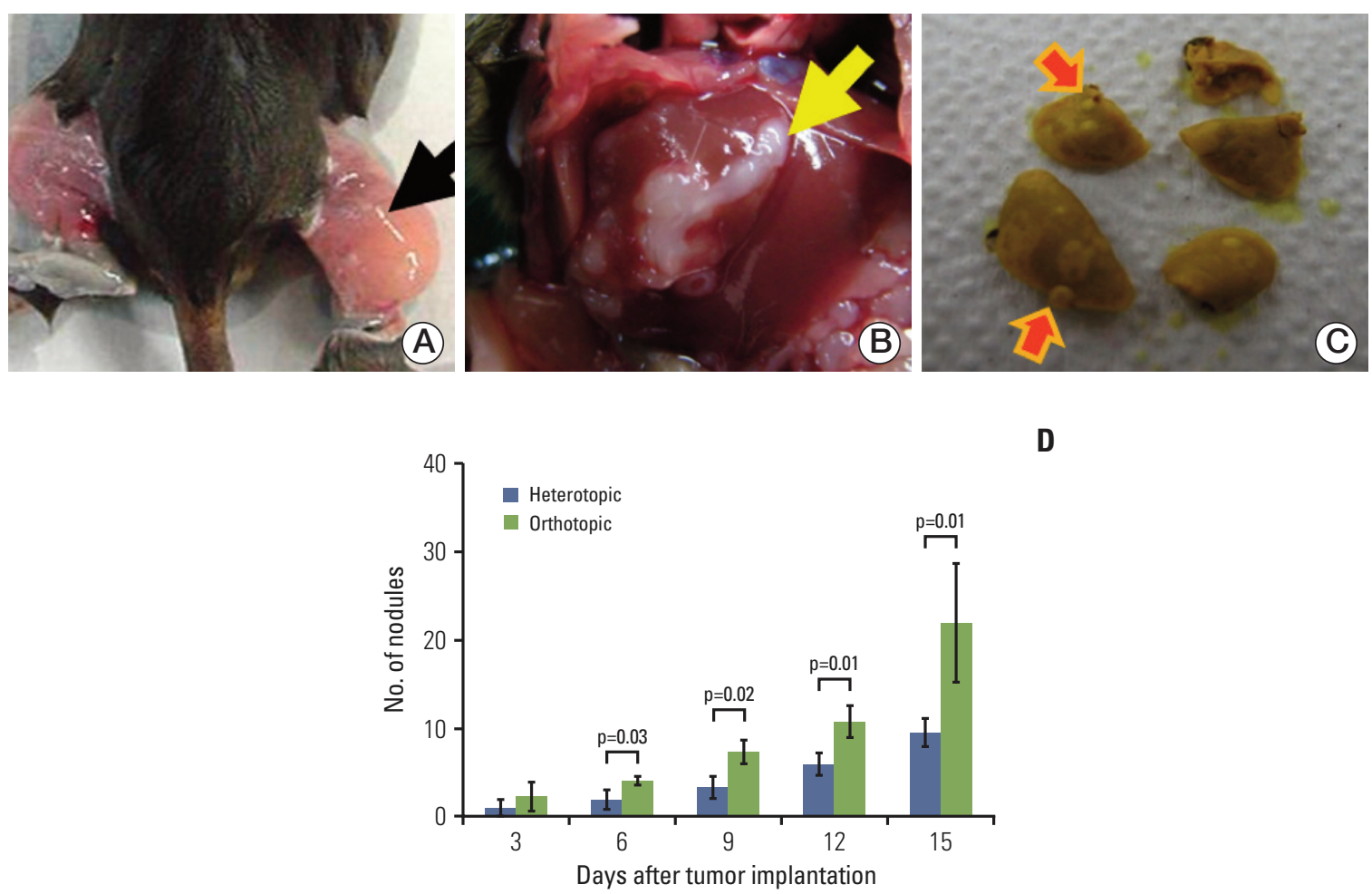

D

Fig. 1. Tumor cells were implanted in the thigh muscle (arrow) in the heterotopic tumor model (A) and at a subcapsular site (arrow) in the liver in the orthotopic tumor model (B). The frequency of lung metastases (arrows) was determined after fixation with Bouin's solution (C) under a light microscope. The number of metastatic lung nodules was higher in the orthotopic tumor model than in the heterotopic tumor model (D). The number of metastatic lung nodules was found to significantly increase in the orthotopic tumor model at $6(\mathrm{p}=0.03), 9(\mathrm{p}=0.02), 12(\mathrm{p}=0.01)$, and 15 days $(\mathrm{p}=0.01)$ after tumor implantation. Groups consisted of six mice each.

vascular endothelial growth factor (VEGF) and CD31 and key regulatory molecules for TME such as cyclooxygenase-2 (COX-2) and transforming growth factor beta1 (TGF- $\beta 1$ ). Immunohistochemical staining procedures were performed from 4- $\mu \mathrm{m}$-thick paraffin cut sections. Microvessel density (MVD) was assessed using the criteria described by Weidner [8]. Areas of highest neovascularization were identified as regions of invasive carcinoma and the highest numbers of discrete microvessels showed staining for CD31.

\section{Western blot analysis of tumor microenvironmental mol- ecules}

The effects of radiation on tumor microenvironmental molecules were analyzed by Western blotting. Tumor tissue was obtained from the heterotopic and orthotopic tumor models, and normal peritumor liver tissue was collected from the orthotopic tumor model. For detection of specific proteins, the following antibodies were used at the concentrations rec- ommended by the manufacturers: a mouse COX-2 monoclonal antibody (Santa Cruz Biotechnology, Santa Cruz, CA), and rabbit polyclonal antibodies to TGF- $\beta 1$ and VEGF (Abcam, Cambridge, MA). After washing in TBST, the membranes containing blotted proteins were incubated for 1 hour at room temperature with either an anti-rabbit (Cell Signaling Technology, Beverly, MA) or anti-mouse (Santa Cruz Biotechnology) immunoglobulin (IgG) secondary antibody, horseradish peroxidase conjugate. Detected proteins were quantified by densitometry (Amersham Pharmacia Biotech, Piscataway, NJ) after chemiluminescence detection (Fuji Photo Film, Tokyo, Japan) using the ECL western blot detection system (Amersham Pharmacia Biotech).

\section{Determination of the host response to different microen- vironments by enzyme-linked immunosorbent assay}

Serum was collected from the orthotopic and heterotopic tumor models for measurement of serum interleukin-6 

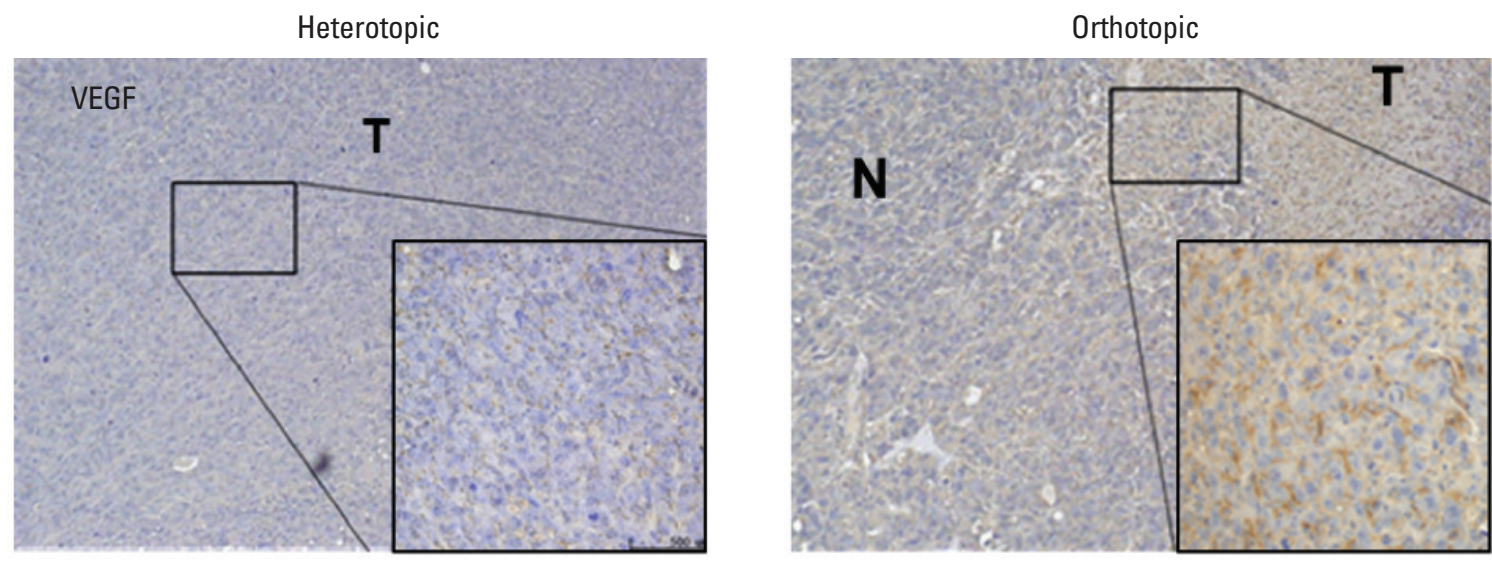

A
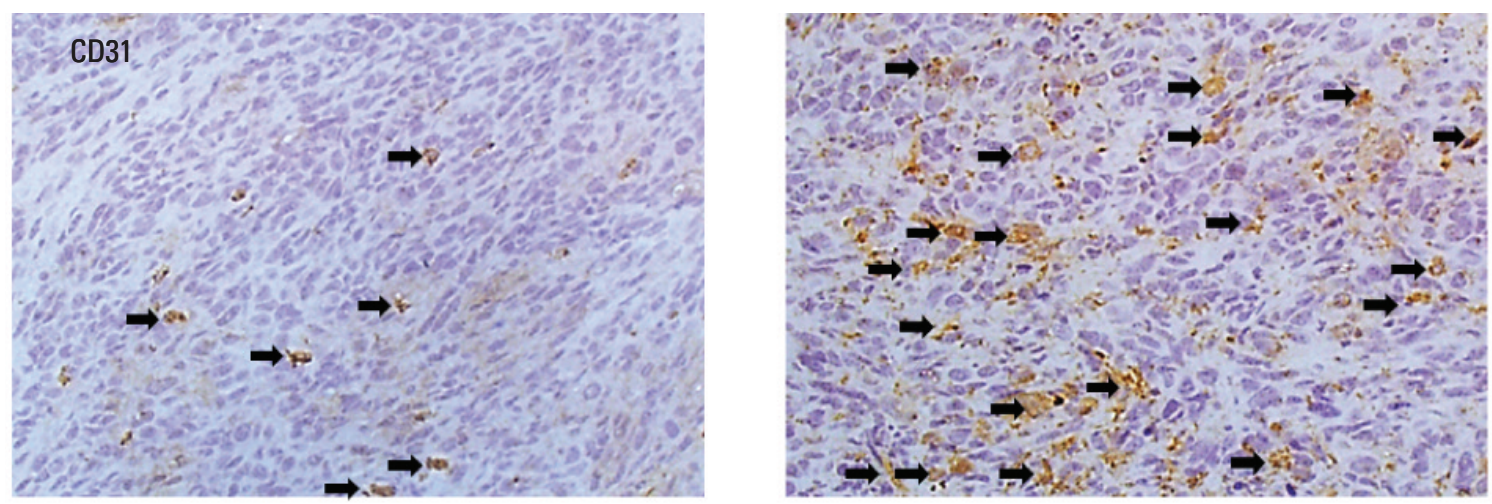

B

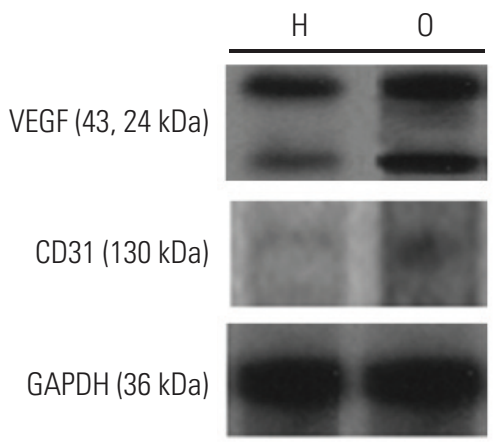

C

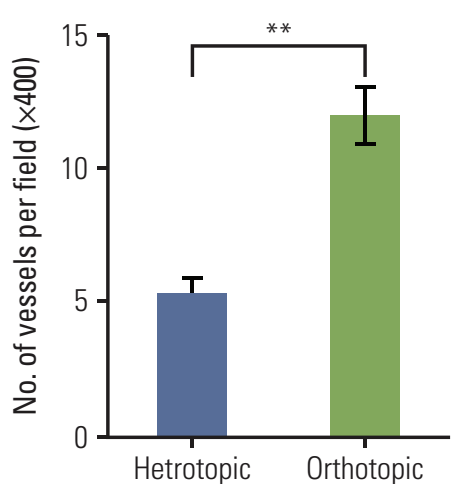

D

Fig. 2. (A) The orthotopic tumor model showed intense cytoplasmic immunoreactivity $(\times 400)$. N, peritumor normal liver; $\mathrm{T}$, tumor tissue. (B) The immunohistochemical stains of CD31 (arrows) for heterotopic and orthotopic tumor model $(\times 400)$. (C) Vascular endothelial growth factor (VEGF) protein levels were elevated in the orthotopic tumor model (O) compared with the heterotopic tumor model $(\mathrm{H})$. CD31 expression was also higher in the orthotopic model than in the heterotopic tumor model. GAPDH, glyceraldehyde 3-phosphate dehydrogenase. (D) Microvessel density using CD31 increased significantly in the orthotopic tumor model $\left({ }^{* *} \mathrm{p}<0.01\right)$. Arrows indicate the microvessels of positive CD31 immunohistochemical staining. The number of mice was six per group.

(IL-6) and VEGF levels by enzyme-linked immunosorbent assay (ELISA) using Quantikine ELISA Kits (R\&D System, Minneapolis, MN) according to the supplier's instructions.
Blood was obtained via closed cardiac puncture using a 22-gauge hypodermic needle and a subxyphoid approach. 


\section{Immune response of the normal peritumor liver and tumor by flow cytometry}

For flow cytometry, the normal peritumor liver and tumor samples were obtained, and spleen tissues were sampled as a control. Flow cytometry was performed by FACS Calibur and FACS Cantoll (BD Biosciences, San Jose, CA), and all antibodies used in this study were purchased as in our previous study [9].

\section{Statistical analysis}

Results are expressed as mean \pm standard error. The $t$ test was used to determine the significance of the differences. All tests were two-sided, and a p-value less than 0.05 indicated statistical significance.

\section{Results}

\section{Establishment of different TME animal models for murine hepatocarcinoma}

Successful tumor formation was achieved in both the heterotopic and orthotopic models after tumor cell inoculation. None of the animals died during inoculation. Features of the heterotopic and orthotopic tumor model after tumor cell implantation are shown in Fig. 1A and B.

Each murine HCa-I showed rapid growth and began to spread to the lung. In comparison of tumor behavior between the heterotopic and orthotopic models, the average number of metastatic lung nodules (Fig. 1C) showed a significant increase in the orthotopic tumor model at 6, 9, 12, and 15 days after tumor implantation $(\mathrm{p}=0.03, \mathrm{p}=0.02, \mathrm{p}=0.01$, and $\mathrm{p}=0.01$, respectively). Fifteen days after tumor implantation, the average numbers of lung nodules in the heterotopic and orthotopic tumor models were $9.7 \pm 2.8$ and $22.0 \pm 11.7$, respectively ( $\mathrm{p}=0.01$ ) (Fig. 1D). Thus, the orthotopic tumor model showed a higher metastatic potential than the heterotopic tumor model.

To investigate the angiogenic factors that prominently contribute to tumor stroma, VEGF expression and MVD were compared in the different TME models. Higher VEGF expression was observed in the orthotopic than in the heterotopic tumor model as determined by immunohistochemical staining. In the orthotopic tumor model, VEGF expression was observed mainly in the tumor area, whereas only scant VEGF expression was observed in the normal pertitumor liver (Fig. 2A). Western blot analysis also showed that VEGF expression was higher in the orthotopic than in the heterotopic tumor model (Fig. 2C).

The MVD in each experimental group was evaluated by immunohistochemical staining for CD31 (Fig. 2B and D). Significantly higher MVD was observed in the orthotopic than in the heterotopic tumor model ( $\mathrm{p}<0.01)$, and results of western blotting showed an increase in CD31 expression in the orthotopic tumor model (Fig. 2D). These data indicate that the orthotopic tumor model might have a higher metastatic potential than the heterotopic tumor model.

For evaluation of TGF- $\beta 1$, key regulatory molecules involved in the TME, the expression and location of TGF- $\beta 1$ protein were determined by immunohistochemistry. High expression of TGF- $\beta 1$ was observed in both the heterotopic and the orthotopic tumor models (Fig. 3A). Interestingly, in the orthotopic tumor model, TGF- $\beta 1$ expression was more prominent in the peripheral tumor area than in the tumor region. Western blot assays showed slightly higher TGF- $\beta 1$ expression in the orthotopic tumor model than in the heterotopic tumor model ( $p>0.05)$ (Fig. 3C).

COX-2 is also a key regulatory molecule in the TME and plays a vital role in inhibiting apoptosis and stimulating tumor angiogenesis. To determine the cellular localization of active COX-2 in each tumor model, frozen sections were stained for COX-2 (Fig. 3B). COX-2 expression was more prominent in the orthotopic tumor area, and only slight staining was detected in the heterotopic tumor tissue. In western blotting analysis, the tumor showed higher COX-2 expression in the orthotopic tumor model than in the heterotopic tumor model (Fig. 3C).

\section{Tumor microenvironmental responses to radiation}

Tumor microenvironmental responses after radiation exposure were evaluated according to different TME models. Irradiated tumor samples were obtained on the third day after radiation. The tumor compartment showed no significant response after radiation in the heterotopic tumor model, because prominent expression was not observed in tumor tissue before radiation (Fig. 4A-D). The orthotopic tumor model had far advanced and metastatic disease, therefore, COX-2, VEGF, and TGF- $\beta 1$ showed high expression before radiation and decreased expression after radiation in tumor tissue (Fig. 4A-C). Results of western blot analysis also showed that COX-2 and TGF- $\beta 1$ expression in tumor tissue of the orthotopic tumor model was slightly down-regulated after radiation except VEGF (Fig. 4D). Although we tried to obtain information about the interaction between the tumor and normal peritumor liver tissue, we found no significant radiation effect due to sparse expression of host tissue.

Local radiotherapy may have an effect on systemic cytokine levels, which may show altered radiation responses in different TMEs; therefore the serum IL-6 level was evalu- 
Heterotopic
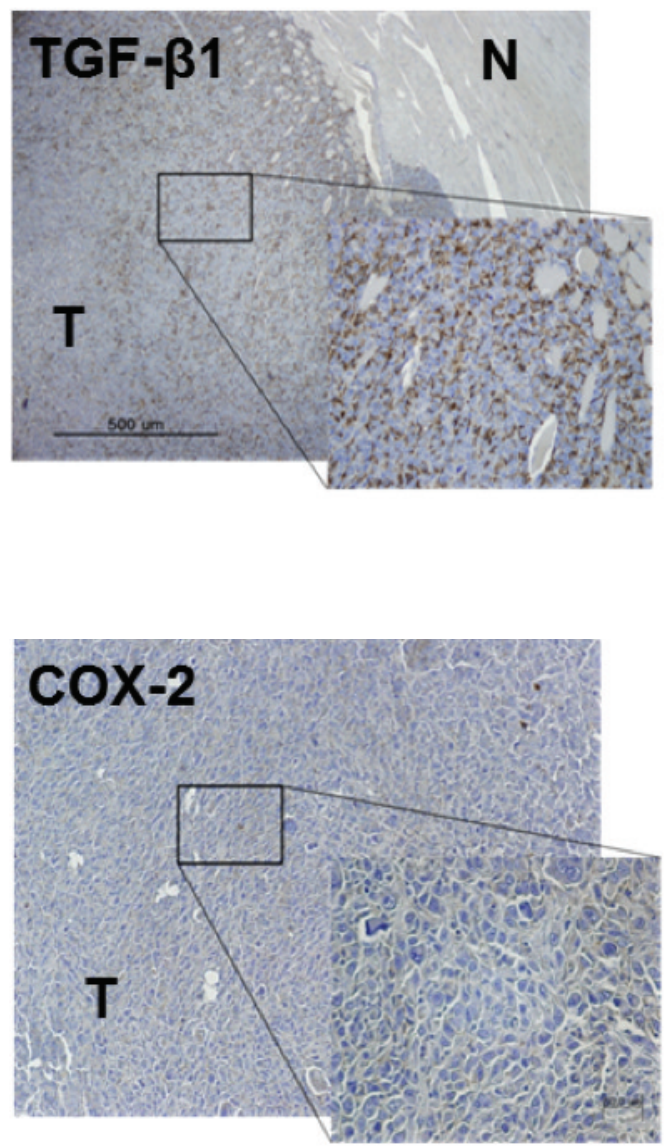

Orthotopic

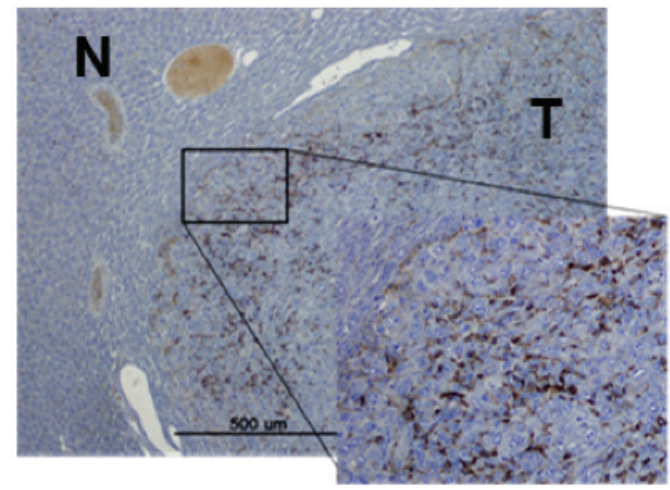

B

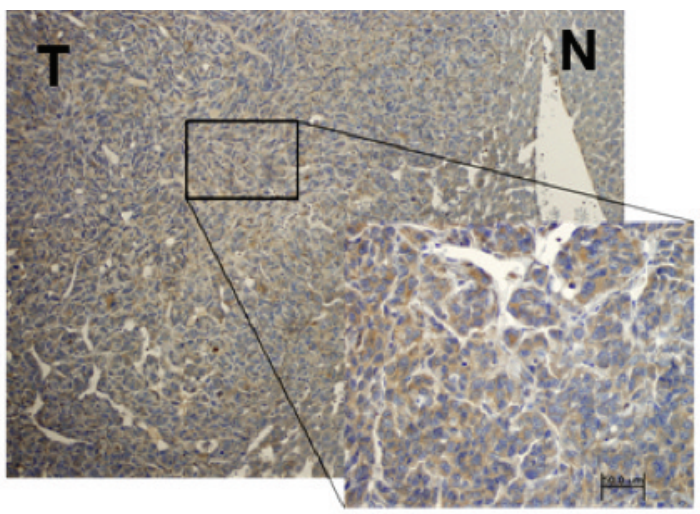

C

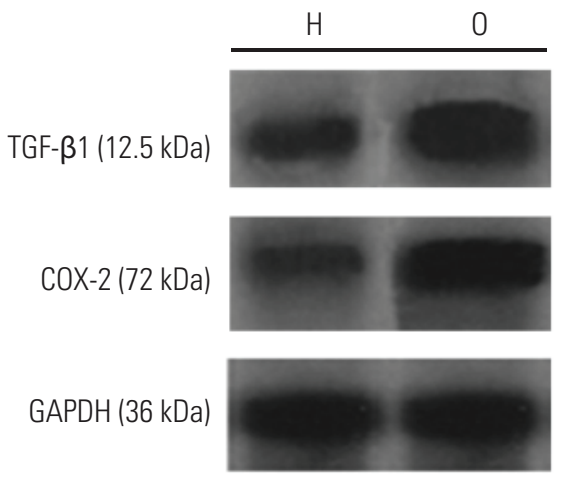

Fig. 3. (A) Orthotopic and heterotopic tumor models demonstrated intense transforming growth factor beta1 (TGF- $\beta 1$ ) immunoreactivity $(\times 400)$. N, peritumor normal liver; $T$, tumor tissue. (B) The orthotopic tumor model showed predominant expression of cyclooxygenase-2 (COX-2) in the cytoplasm of peripheral tumor tissue $(\times 400)$. (C) Western blot assays using antibodies against TGF- $\beta 1$ and COX-2 in the orthotopic $(\mathrm{O})$ and heterotopic $(\mathrm{H})$ tumor models showed higher TGF- $\beta 1$ and COX-2 expression in the orthotopic model than in the heterotopic tumor model. GAPDH, glyceraldehyde 3-phosphate dehydrogenase. 

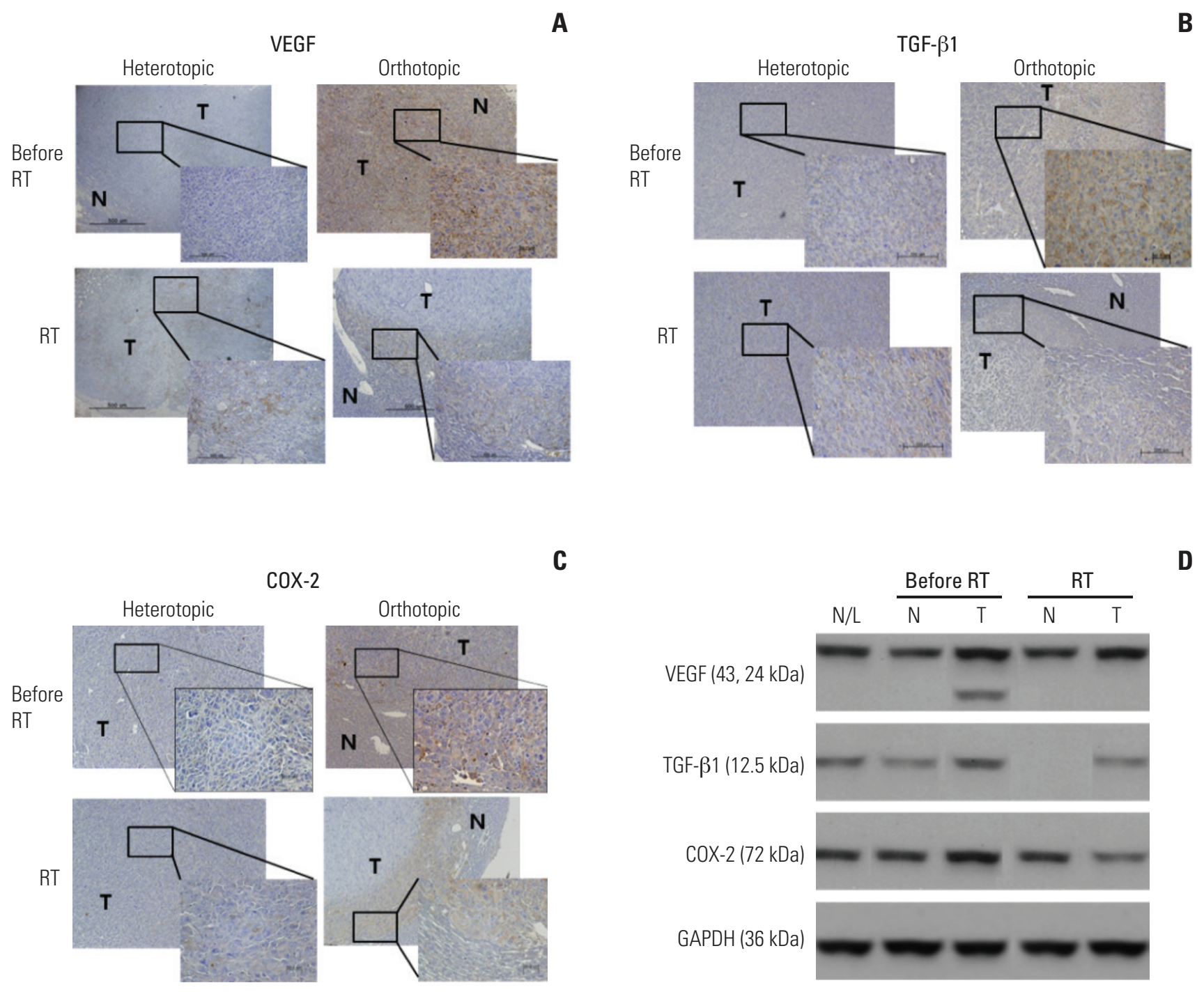

C

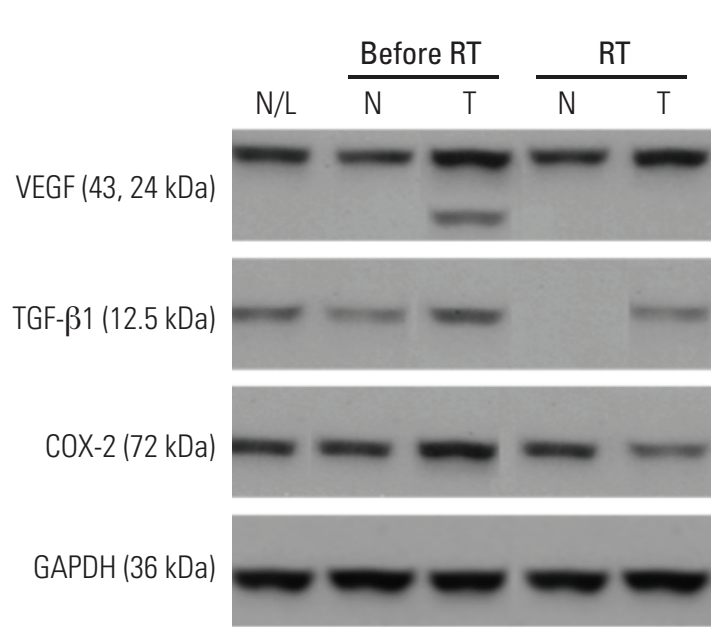

Fig. 4. Immunohistochemical staining with antibodies against vascular endothelial growth factor (VEGF), transforming growth factor beta1 (TGF- $\beta 1$ ), and cyclooxygenase-2 (COX-2) in the heterotopic and orthotopic tumor models (A-C). After radiation, staining for VEGF, TGF- $\beta 1$, and COX-2 decreased in the orthotopic tumor model $(\times 400)$, while the tumor compartment in the heterotopic tumor model showed no significant responses after radiation ( 3 days after radiation). (D) These findings were confirmed in the orthotopic tumor model by western blot assay. Expression of VEGF, TGF- $\beta 1$, and COX-2 in tumor tissues decreased after radiation, not in peritumoral normal tissue. (Continued to the next page)

ated (Fig. 4E). IL-6 elevation after radiation was observed in heterotopic and orthotopic tumor models $(\mathrm{p}=0.01$ and $\mathrm{p}=0.01$, respectively), and the orthotopic tumor model showed a significantly higher level of IL-6 than the heterotopic tumor model $(p<0.01)$. Higher serum VEGF levels were also measured in the orthotopic model than in the heterotopic tumor model and the VEGF level was reduced after irradiation in the orthotopic tumor model, but the difference was not statistically significant (Fig. 4F).

\section{Immune cell expression after radiation}

Immune cells were analyzed according to different TME, and we observed the elevation of frequency of regulatory $\mathrm{T}$ cell (Treg) cells and increased population of $\mathrm{CD} 11 \mathrm{~b}^{+} \mathrm{Gr}-1^{+}$ myeloid-derived suppressor cells (MDSCs) in heterotopic and orthotopic tumor models. $\mathrm{CD} 11 \mathrm{~b}^{+} \mathrm{Gr}-1^{+}$MDSCs are known as important other immune response regulators in the TME. In particular, the orthotopic tumor model showed 

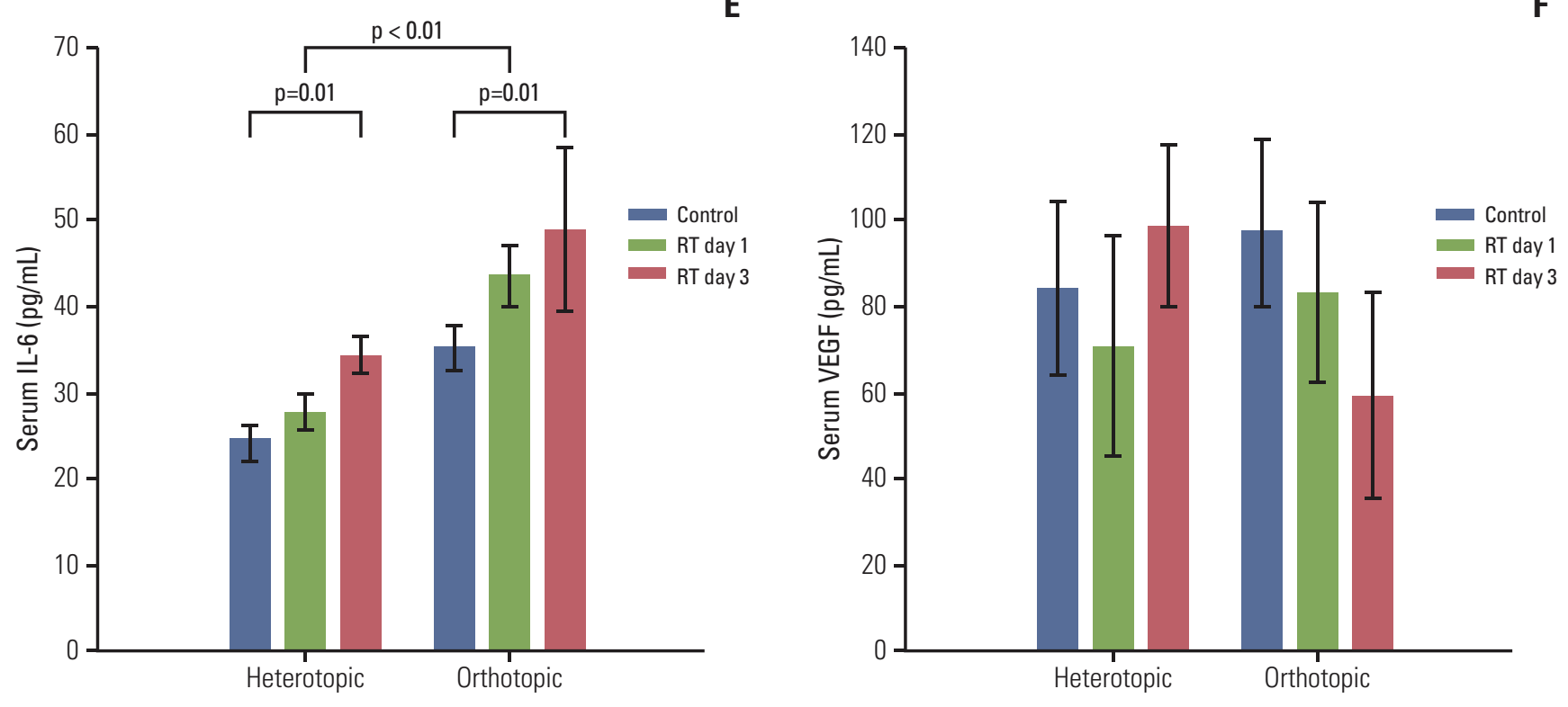

Fig. 4. (Continued from the previous page) (E) Serum interleukin-6 (IL-6) increased after irradiation in heterotopic and orthotopic tumor models ( $\mathrm{p}=0.01$ and $\mathrm{p}=0.01$ ), and the orthotopic tumor model showed a significantly higher level of IL- 6 than the heterotopic tumor model $(\mathrm{p}<0.01)$. Increased serum VEGF levels were also measured in the orthotopic tumor model and this level was reduced after irradiation in the orthotopic tumor model, but the difference was not significant (F). The sampling number was six per group. N/L, normal liver of naïve mouse; $\mathrm{N}$, normal peritumor liver; $\mathrm{T}$, tumor; $\mathrm{RT}$, radiation treatment; GAPDH, glyceraldehyde 3-phosphate dehydrogenase.

a significantly higher frequency of $\mathrm{CD} 11 \mathrm{~b}^{+} \mathrm{Gr}-1^{+}$MDSCs than the heterotopic tumor model [9].

To further investigate the frequency of immune cells in orthotopic tumor microenvironmental immune cells and their response after irradiation, expression of $\mathrm{CD} 4^{+}, \mathrm{CD} 8^{+}$, CD25 ${ }^{+}$Foxp3 Treg cells, and CD11b ${ }^{+} \mathrm{Gr}-1^{+}$MDSCs was examined in the orthotopic tumor bearing mouse model. The frequencies of total CD4 and CD8 T cells in tumor-infiltrating lymphocytes (TILs) are shown in the irradiated and nonirradiated orthotopic tumor model (Fig. 5A). The number of these cells showed a slight decrease after radiation, although it was not statistically meaningful. The number of $\mathrm{CD} 25^{+}$ Foxp3 Treg cells in the tumor was significantly decreased after radiation (Fig. 5B), and CD11 $\mathrm{b}^{+} \mathrm{Gr}-\mathrm{1}^{+}$MDSCs expression was also significantly decreased after radiation (Fig. 5C).

\section{Discussion}

Histological examination of tumors shows the presence of many non-epithelial cell types, which comprise the tumor stroma. This suggests that the TME is not defined only by the properties of the malignant cell population but rather is a product of the interaction between tumor and non-neoplastic tissue. In this light, heterotopic tumor implantation has a limitation for use in investigation of the nature of human cancers and their therapeutic approaches. Gao et al. [10] established a nude mouse model of human hepatocellular carcinoma (HCC) via orthotopic implantation of histologically intact tissue. Various orthotopic HCC models have been established in nude mice; the current study established a syngenic orthotopic tumor model for evaluation of microenvironmental alterations, including immunologic responses. This model also represents the features of a mouse model with murine HCC and could be an interesting tool for use in exploration of therapeutic strategies of HCC and its microenvironmental effects.

According to several research studies, heterotopic and orthotopic tumors differ in terms of both angiogenesis and metastasis. Morikawa et al. [11] demonstrated that implantation of KM12 cells into the cecal wall (orthotopic) or a subcutaneous site (heterotopic) in nude mice produces tumors with different metastatic potentials: orthotopic tumors are invasive and metastatic, whereas heterotopic tumors are not, 

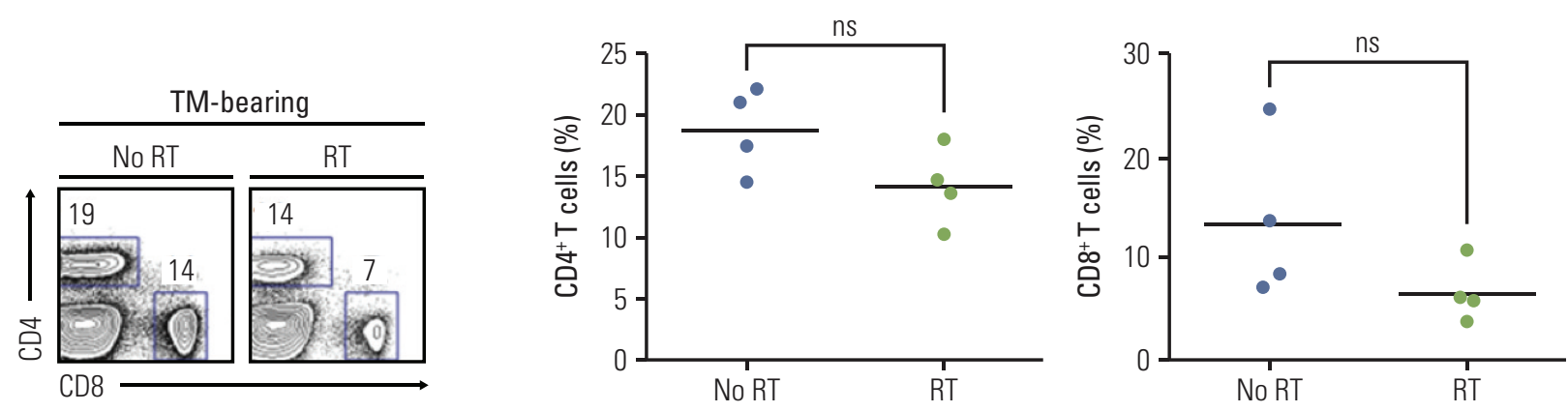

A
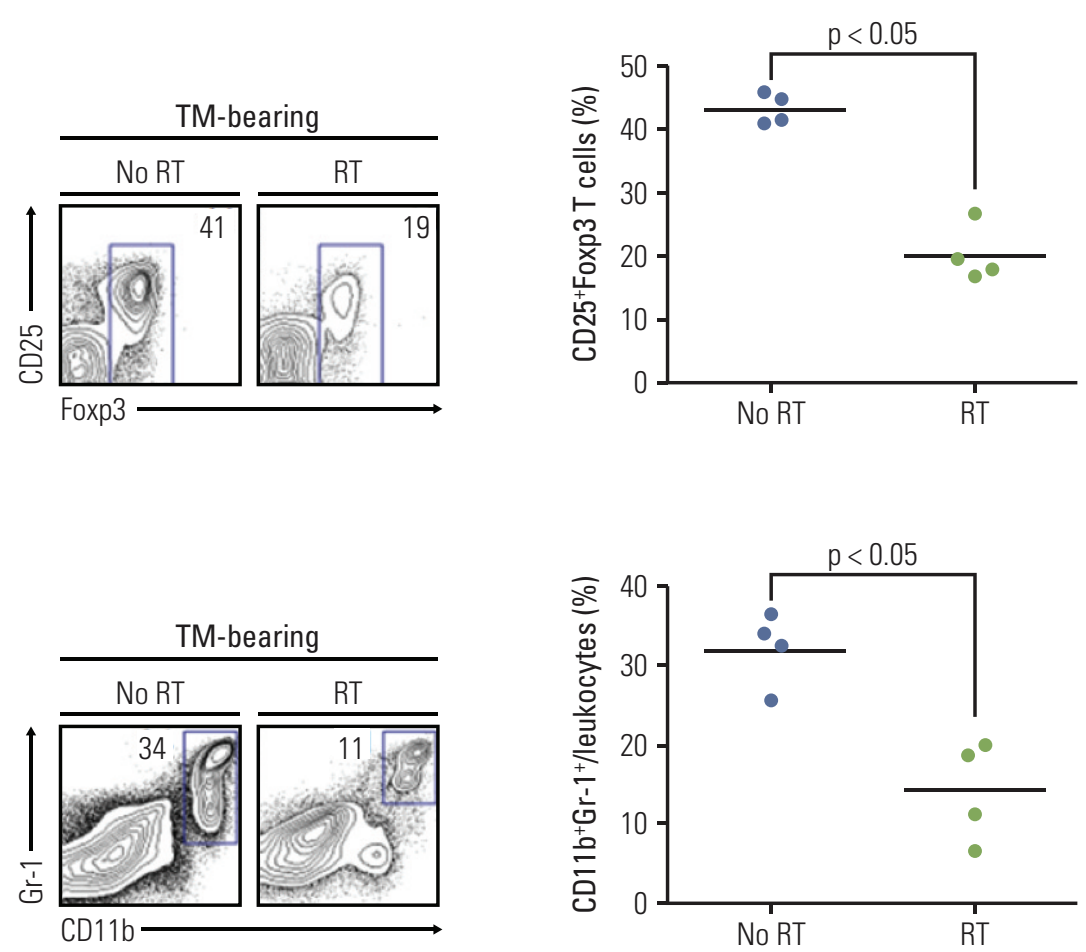

Fig. 5. Immune cell expression after radiation ( $\mathrm{n}=4$ mice per each group). Irradiated orthotopic tumor samples were obtained on the third day after radiation. (A) The frequency of total CD4 and CD8 T cells in tumor-infiltrating lymphocytes are shown in the irradiated and non-irradiated orthotopic tumor model. In tumor bearing mice (TM-bearing) mice, the frequency of CD4 and CD8 T cells showed no significant difference after radiation. (B) The number of CD25 ${ }^{+}$Foxp3 Treg cells in the tumor was significantly decreased after radiation $(\mathrm{p}<0.05)$. (C) Myeloid-derived suppressor cell $\mathrm{CD} 11 \mathrm{~b}^{+} \mathrm{Gr}-1^{+}$expression was also significantly decreased after radiation $(\mathrm{p}<0.05)$. Treg, regulatory $\mathrm{T}$ cell; RT, radiation treatment; $\mathrm{ns}$, not significant.

suggesting that different organ environments may differentially influence the expression of metastasis-related genes. Onogawa et al. [12] reported that levels of epidermal growth factor receptor, basic fibroblast growth factor, IL-8, VEGF-C, and VEGF-D protein were higher in orthotopic than in heterotopic tumors. In our current study, a more aggressive potential for tumor growth and lung metastasis was obse- rved for the orthotopic model than for the heterotopic tumor model. In addition, angiogenic factors (VEGF and CD31), key regulatory molecules (COX-2 and TGF- $\beta 1$ ), and serum cytokine (IL-6) increased in the orthotopic model compared with that in the heterotopic tumor model. Lung metastasis may therefore depend on changes in the TME or on tumor cell-intrinsic genetic events. One might conclude that hetero- 
topic tumors require a bit more time to reach the same number of lung metastases. Therefore, the heterotopic tumor model is also valuable for investigating the metastatic process or potential. However, there was still a lack of experimental studies regarding the differences between primary tumors and late-stage metastatic disease.

Several clinical studies have demonstrated a direct correlation of high primary tumor MVDs with increased incidence of metastases, and tumor MVD was a significant and independent prognostic indicator for relapse-free and overall survival of cancer patients [13]. Similarly, an elevated tumor or serum level of IL- 6 was associated with increased incidence of metastasis in cancer patients $[14,15]$. In our tumor model, angiogenic factors (VEGF and CD31) were also higher in the orthotopic model than in the heterotopic tumor model. These findings suggested that VEGF expression might be associated with an increased lung metastatic potential in the orthotopic tumor model compared with the heterotopic tumor model. IL-6 is involved in the immune response, inflammation, and hematopoiesis. Mouthon et al. [16] found that IL-6 was increased in plasma from intestine- and liver-irradiated mice. Chou et al. [17] reported a nonspecific increase in IL-6 levels in patients undergoing radiotherapy in a part of the liver. IL-6 is also regarded as a major trigger of VEGF induction, and the mechanism of VEGF induction by IL- 6 may be critically involved in the progression of tumor cell growth [18]. A significantly higher level of IL-6 was observed in our orthotopic tumor model than the heterotopic tumor model, and the irradiated orthotopic tumor model showed increased serum IL-6 levels.

Considering the TME in controlling metastasis and the complexity of the various TME compartments, the orthotopic animal model is a useful tool for evaluation of immunologic findings due to its retention of a similar natural environment. Pang et al. [19] reported that the representation of $\mathrm{CD} 8^{+} \mathrm{T}$ cells, natural killer (NK) cells, NK-T cells, and gamma delta $(\gamma \delta) \mathrm{T}$ cells was significantly reduced in TILs in HCC patients. The $\mathrm{CD} 4^{+} \mathrm{T}$ population was substantially expanded in TILs, and a significant increase in CD25 ${ }^{+}$Foxp3 Treg cells was observed in the tumor tissue [19]. Our previous study demonstrated an increased population of Treg cells and up-regulation of inhibitory receptors by Treg cells in TILs from heterotopic and orthotopic tumor models [9]. In addition, CD11 b ${ }^{+} \mathrm{Gr}-1^{+}$MDSCs also increased in both tumor models. MDSCs expressing $\mathrm{CD} 11 \mathrm{~b}^{+} \mathrm{Gr}-\mathrm{1}^{+}$are known to be a negative regulator of immune responses in cancer and other diseases. MDSCs are also associated with the induction of Tregs in the TME, which could produce IL-10 through the activity of the transcription factor Foxp3 [20]. Interestingly, in our current study, orthotopic tumor models showed decreased CD25 $5^{+}$Foxp3 Treg cells and CD $11 b^{+} \mathrm{Gr}-1^{+}$MDSCs in TIL after radiation. These findings suggest that decreased
$\mathrm{CD} 11 \mathrm{~b}^{+} \mathrm{Gr}-1^{+}$MDSCs may enhance immune responses and ameliorate radiotherapeutic responses.

Although orthotopic tumor models showed a decrease of CD25 $5^{+}$Foxp3 Treg cells in TIL after radiation in the current study, there are resistant $\mathrm{CD} 25^{+} \mathrm{Foxp} 3$ Treg cells [21]. Therefore, the strategy employing radiotherapy combined with ablation of Treg cells may improve the outcome of treatment and prolong the survival [22]. With the development of molecular target therapies, the use of several drugs, antibodies, or fusion proteins to reduce or eliminate human suppressor cells is also currently under clinical investigation $[23,24]$. Thus, there is considerable support for a rationale for potential clinical trials employing multimodal TME-based therapies with radiation [25]. These results highlight the potential importance of innate immunity in antitumor responses; further exploration of this system is needed.

\section{Conclusion}

The orthotopic tumor model demonstrated higher lung metastatic potential and more aggressive molecular features (VEGF, COX-2, and TGF- $\beta 1$ ) than the heterotopic tumor model. In the orthotopic tumor model, the angiogenic factor VEGF, key regulatory molecules (TGF- $\beta 1$ and COX-2), and immune cells (CD25 $5^{+}$Foxp3 Treg and $\mathrm{CD} 11 \mathrm{~b}^{+} \mathrm{Gr}-1^{+}$cells) decreased after radiation. These findings suggest that an orthotopic tumor mouse model may be more reflective of the TME and suitable for the investigation of radiation treatment.

\section{Conflicts of Interest}

Conflict of interest relevant to this article was not reported.

\section{Acknowledgments}

This work was supported by the Basic Science Research Program (Grant number; 331-2007-1-E00163) and the Midcareer Researcher Program (NRF-2014R1A2A1A11054463) through the National Research Foundation of Korea funded by the Ministry of Science, ICT \& Future Planning. 


\section{References}

1. Weinberg RA. The biology of cancer. New York: Garland Science; 2007.

2. Oppenheimer SB. Cellular basis of cancer metastasis: a review of fundamentals and new advances. Acta Histochem. 2006; 108:327-34.

3. Cretu A, Brooks PC. Impact of the non-cellular tumor microenvironment on metastasis: potential therapeutic and imaging opportunities. J Cell Physiol. 2007;213:391-402.

4. Tofilon PJ, Basic I, Milas L. Prediction of in vivo tumor response to chemotherapeutic agents by the in vitro sister chromatid exchange assay. Cancer Res. 1985;45:2025-30.

5. Meyn RE, Stephens LC, Ang KK, Hunter NR, Brock WA, Milas $\mathrm{L}$, et al. Heterogeneity in the development of apoptosis in irradiated murine tumours of different histologies. Int J Radiat Biol. 1993;64:583-91.

6. Lee EJ, Park HJ, Lee IJ, Kim WW, Ha SJ, Suh YG, et al. Inhibition of IL-17A suppresses enhanced-tumor growth in low dose pre-irradiated tumor beds. PLoS One. 2014;9:e106423.

7. Kim W, Seong J, An JH, Oh HJ. Enhancement of tumor radioresponse by wortmannin in $\mathrm{C} 3 \mathrm{H} / \mathrm{HeJ}$ hepatocarcinoma. J Radiat Res. 2007;48:187-95.

8. Weidner N. Current pathologic methods for measuring intratumoral microvessel density within breast carcinoma and other solid tumors. Breast Cancer Res Treat. 1995;36:169-80.

9. Park HJ, Kusnadi A, Lee EJ, Kim WW, Cho BC, Lee IJ, et al. Tumor-infiltrating regulatory $\mathrm{T}$ cells delineated by upregulation of PD-1 and inhibitory receptors. Cell Immunol. 2012; 278:76-83.

10. Gao YS, Chen XP, Li KY, Wu ZD. Nude mice model of human hepatocellular carcinoma via orthotopic implantation of histologically intact tissue. World J Gastroenterol. 2004;10: 3107-11.

11. Morikawa K, Walker SM, Jessup JM, Fidler IJ. In vivo selection of highly metastatic cells from surgical specimens of different primary human colon carcinomas implanted into nude mice. Cancer Res. 1988;48:1943-8.

12. Onogawa S, Kitadai Y, Tanaka S, Kuwai T, Kuroda T, Chayama K. Regulation of vascular endothelial growth factor (VEGF)-C and VEGF-D expression by the organ microenvironment in human colon carcinoma. Eur J Cancer. 2004;40: 1604-9.

13. Benoy IH, Salgado R, Elst H, Van Dam P, Weyler J, Van Marck $\mathrm{E}$, et al. Relative microvessel area of the primary tumour, and not lymph node status, predicts the presence of bone marrow micrometastases detected by reverse transcriptase polymerase chain reaction in patients with clinically non-metastatic breast cancer. Breast Cancer Res. 2005;7:R210-9.

14. Guang-Wu H, Sunagawa M, Jie-En L, Shimada S, Gang Z, Tokeshi $Y$, et al. The relationship between microvessel density, the expression of vascular endothelial growth factor (VEGF), and the extension of nasopharyngeal carcinoma. Laryngoscope. 2000;110:2066-9.

15. Huang SP, Wu MS, Wang HP, Yang CS, Kuo ML, Lin JT. Correlation between serum levels of interleukin- 6 and vascular endothelial growth factor in gastric carcinoma. J Gastroenterol Hepatol. 2002;17:1165-9.

16. Mouthon MA, Vandamme M, van der Meeren A, Gourmelon $\mathrm{P}$, Gaugler MH. Inflammatory response to abdominal irradiation stimulates hemopoiesis. Int J Radiat Biol. 2001;77:95-103.

17. Chou CH, Chen PJ, Jeng YM, Cheng AL, Huang LR, Cheng JC. Synergistic effect of radiation and interleukin- 6 on hepatitis $B$ virus reactivation in liver through STAT3 signaling pathway. Int J Radiat Oncol Biol Phys. 2009;75:1545-52.

18. Wei LH, Kuo ML, Chen CA, Chou CH, Lai KB, Lee CN, et al. Interleukin-6 promotes cervical tumor growth by VEGFdependent angiogenesis via a STAT3 pathway. Oncogene. 2003;22:1517-27.

19. Pang YL, Zhang HG, Peng JR, Pang XW, Yu S, Xing Q, et al. The immunosuppressive tumor microenvironment in hepatocellular carcinoma. Cancer Immunol Immunother. 2009;58: 877-86.

20. Saraiva M, O'Garra A. The regulation of IL-10 production by immune cells. Nat Rev Immunol. 2010;10:170-81.

21. Komatsu N, Hori S. Full restoration of peripheral Foxp3+ regulatory $\mathrm{T}$ cell pool by radioresistant host cells in scurfy bone marrow chimeras. Proc Natl Acad Sci U S A. 2007;104:8959-64.

22. Bos PD, Plitas G, Rudra D, Lee SY, Rudensky AY. Transient regulatory $\mathrm{T}$ cell ablation deters oncogene-driven breast cancer and enhances radiotherapy. J Exp Med. 2013;210:2435-66.

23. Rech AJ, Vonderheide RH. Clinical use of anti-CD25 antibody daclizumab to enhance immune responses to tumor antigen vaccination by targeting regulatory T cells. Ann N Y Acad Sci. 2009;1174:99-106.

24. Saha A, Chatterjee SK. Combination of CTL-associated antigen- 4 blockade and depletion of CD25 regulatory T cells enhance tumour immunity of dendritic cell-based vaccine in a mouse model of colon cancer. Scand J Immunol. 2010;71: 70-82.

25. Deng L, Liang H, Burnette B, Beckett M, Darga T, Weichselbaum RR, et al. Irradiation and anti-PD-L1 treatment synergistically promote antitumor immunity in mice. J Clin Invest. 2014;124:687-95. 